Check for updates

Cite this: J. Mater. Chem. B, 2020, 8, 7289

DOI: $10.1039 / d 0 t b 90127 j$

rsc.li/materials-b

\section{Correction: Potent and regularizable crosslinking of ultrafine fibrous protein scaffolds for tissue engineering using a cytocompatible disaccharide derivative}

\author{
Helan $\mathrm{Xu}^{\text {a }}$ Peng Liu, ${ }^{\mathrm{b}}$ Xiang $\mathrm{Mi}^{\mathrm{b}}{ }^{\mathrm{L}}$ Lan $\mathrm{Xu}^{\mathrm{c}}$ and Yiqi Yang*ade
}

Correction for 'Potent and regularizable crosslinking of ultrafine fibrous protein scaffolds for tissue engineering using a cytocompatible disaccharide derivative' by Helan Xu et al., J. Mater. Chem. B, 2015, 3, 3609-3616, DOI: 10.1039/C4TB02100B.

The authors regret mistakes in their manuscript. This paper shared some contents with another paper composed by the same authors in Macromolecular Materials and Engineering, ${ }^{1}$ which should have been cited in this J. Mater. Chem. B paper.

The following results were from our experiments and are shared in both papers.

1. Part of Fig. 2 in this J. Mater. Chem. B paper, i.e., CLSM images of zein samples with and without OS crosslinking, were adapted from Fig. 3 in ref. 1.

2. Part of Fig. 3ii in this paper, i.e., SDS-PAGE of zein crosslinked by OS, was adapted from Fig. 4 a in ref. 1 .

3. Part of Fig. 8 in this paper, i.e., cell images from zein scaffold crosslinked by OS, was adapted from Fig. 8 in ref. 1.

The partial duplication of figures was because the same oxidized sucrose (OS) treated samples were used for comparison with citric acid treated samples in this J. Mater. Chem. B paper and glutaraldehyde treated samples in ref. 1. However, both papers are fundamentally different. In the J. Mater. Chem. B paper, we compared OS with citric acid, a safe crosslinker with acceptable crosslinking capability. Results demonstrated that OS was as safe as, but more effective than citric acid. In ref. 1, we compared OS with glutaraldehyde, an effective but highly toxic crosslinker. Results verified that OS was as effective as, but much safer than glutaraldehyde.

The authors regret some errors that are corrected as below.

1. One concentration in the caption of Fig. 2 was reported incorrectly as "...5 wt\% of OS. ..".

The corrected figure caption is "CLSM images of the top $45^{\circ}$ view (fourth column) of the electrospun 3D ultrafine fibrous zein scaffolds without crosslinking, 8 wt $\%$ of OS and 10 wt $\%$ of CA.".

2. One concentration in the caption of Fig. 3ii was reported incorrectly as "...2 wt\% (lane B)...".

The corrected figure caption is "(ii) SDS-PAGE of, lane A, standard protein marker; zein crosslinked with OS at concentrations of $1 \mathrm{wt} \%$ (lane B); $3 \mathrm{wt} \%$ (lane C); $5 \mathrm{wt} \%$ (lane D); $8 \mathrm{wt} \%$ (lane E); $10 \mathrm{wt} \%$ (lane F)."

3. One sample name in the caption of Fig. 8 was reported incorrectly as "...glutaraldehyde...".

The corrected figure caption is "Spreading of MC 3T3 cells on the plane at the depth of $45 \mu \mathrm{m}$ in the electrospun 3D ultrafine fibrous zein scaffolds crosslinked with oxidized sucrose and CA after culturing for 7 days."

4. In the main text of p. 3612 , the concentration of OS was incorrectly reported as "8 wt\%" in the sentence "The ${ }^{1} \mathrm{H}-\mathrm{NMR}$ spectra of uncrosslinked zein and 8 wt $\%$ OS crosslinked zein are illustrated in Fig. 4".

The corrected sentence is "The ${ }^{1} \mathrm{H}-\mathrm{NMR}$ spectra of uncrosslinked zein and $5 \mathrm{wt} \%$ OS crosslinked zein are illustrated in Fig. 4". 5. The second left bar in the bar graph of Fig. 3iii was incorrectly marked as " $2 \%$ OS".

\footnotetext{
${ }^{a}$ Department of Textiles, Merchandising and Fashion Design, 234, HECO Building, University of Nebraska-Lincoln, Lincoln, NE 68583-0802, USA. E-mail: yyang2@unl.edu; Fax: +1402472 0640; Tel: +14024725197

${ }^{b}$ Key Laboratory of Science and Technology of Eco-Textiles, Ministry of Education, Donghua University, Shanghai 201620, China

${ }^{c}$ Department of Agronomy and Horticulture, University of Nebraska-Lincoln, 279 Plant Science Hall, Lincoln, NE 68583-0915, USA

${ }^{d}$ Department of Biological Systems Engineering, 234, HECO Building, University of Nebraska-Lincoln, Lincoln, NE 68583-0802, USA

${ }^{e}$ Nebraska Center for Materials and Nanoscience, 234, HECO Building, University of Nebraska-Lincoln, Lincoln, NE 68583-0802, USA
} 
The correct OS concentration is " $1 \%$ OS". The bar graph has been modified as shown in the corrected Fig. 3 below.
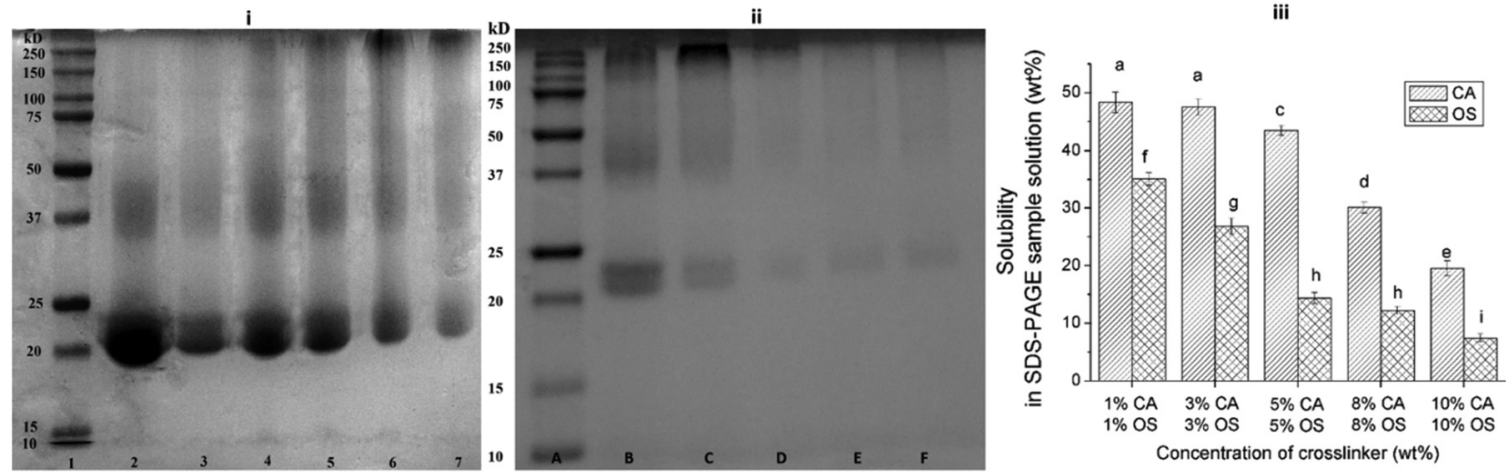

Fig. 3 (i) SDS-PAGE of, lane 1, standard protein marker; lane 2, uncrosslinked zein; zein crosslinked with CA at concentrations of 1 wt\% (lane 3); 3 wt\% (lane 4); $5 \mathrm{wt} \%$ (lane 5); $8 \mathrm{wt} \%$ (lane 6); $10 \mathrm{wt} \%$ (lane 7). (ii) SDS-PAGE of, lane A, standard protein marker; zein crosslinked with OS at concentrations of 2 wt\% (lane B); 3 wt\% (lane C); 5 wt\% (lane D); 8 wt\% (lane E); 10 wt\% (lane F). (iii) Solubility of the samples in (i) and (ii) in the SDS-PAGE sample buffer. Different letters in the figure indicated significant differences. All the crosslinking reactions were carried out under $150{ }^{\circ} \mathrm{C}$ for $2 \mathrm{~h}$.

The Royal Society of Chemistry apologises for these errors and any consequent inconvenience to authors and readers.

\section{References}

1 P. Liu, H. Xu, X. Mi, L. Xu and Y. Yang, Macromol. Mater. Eng., 2015, 300, 414-422. 